\title{
ON THE NASH-MOSER IMPLICIT FUNCTION THEOREM
}

\author{
LARS HÖRMANDER
}

In [1] a general implicit function theorem of Moser's type was derived from the methods of Nash [2]. However, it turns out that better results and simpler proofs may be obtained by a simple modification of this approach combined with standard non-linear functional analysis. We shall present this modification here, choosing this time an absiract setting as for example in Zehnder [3].

Let $E_{a}, a \geqq 0$, be a decreasing family of Banach spaces with injections $E_{b} \subset E_{a}$ of norm $\leqq 1$ when $b \geqq a$. Set $E_{\infty}=\cap E_{a}$ with the weakest topology making the injections $E_{\infty} \subset E_{a}$ continuous, and assume that we have given linear operators $S_{\theta}: E_{0} \rightarrow E_{\infty}$ for $\theta \geqq 1$, such that with constants $C$ bounded, when $a$ and $b$ are bounded,

$$
\begin{gathered}
\left\|S_{\theta} u\right\|_{b} \leqq C\|u\|_{a}, \quad b \leqq a ; \\
\left\|S_{\theta} u\right\|_{b} \leqq C \theta^{b-a}\|u\|_{a}, a<b ; \\
\left\|u-S_{\theta} u\right\|_{b} \leqq C \theta^{b-a}\|u\|_{a}, a>b ; \\
\left\|\frac{d}{d \theta} S_{\theta} u\right\|_{b} \leqq C \theta^{b-a-1}\|u\|_{a} .
\end{gathered}
$$

Hölder spaces are classical examples (see [1, appendix]). The property (iv) is the strongest one; integration of (iv) from $\theta$ to $\infty$ gives (iii) and integration from 1 to $\theta$ gives (ii) (although the constants may become large as $b$ approaches $a$ ). From (ii) and (iii) we obtain the logarithmic convexity of the norms

$$
\|u\|_{\lambda a+(1-\lambda) b} \leqq C\|u\|_{a}^{\lambda}\|u\|_{b}^{1-\lambda} \quad \text { if } \quad 0<\lambda<1 .
$$

In fact, if $c=\lambda a+(1-\lambda) b$ and $a<b$, we obtain from (ii) and (iii)

$$
\|u\|_{c} \leqq\left\|S_{\theta} u\right\|_{c}+\left\|u-S_{\theta} u\right\|_{c} \leqq C\left(\theta^{c-a}\|u\|_{a}+\theta^{c-b}\|u\|_{b}\right) .
$$

Since $\|u\|_{b} \geqq\|u\|_{a}$ the two terms are equal for some $\theta \geqq 1$, which gives (v) with the constant $2 C$.

Condition (iv) provides a convenient way of making a continuous decomposition of an arbitrary $u \in E_{a}$. However, we prefer to use discrete decompositions in order to have no problems with vector valued integration. We therefore choose a fixed sequence with $1=\theta_{0}<\theta_{1}<\ldots \rightarrow \infty$ such that $\theta_{j+i} / \theta_{j}$ is bounded, set $\Delta_{j}=\theta_{j+1}-\theta_{j}$ 
and introduce

$$
R_{j} u=\left(S_{\theta_{j+1}} u-S_{\theta_{j}} u\right) / \Delta_{j} \quad \text { if } \quad j>0, \quad R_{0} u=S_{\theta_{1}} u / \Delta_{0} .
$$

Then we have by (iii)

$$
u=\sum_{0}^{\infty} \Delta_{j} R_{j} u
$$

with convergence in $E_{a}$, if $u \in E_{b}$ for some $b>a$, and (iv) gives for all $b$

$$
\left\|R_{j} u\right\|_{b} \leqq C_{a, b} \theta_{j}^{b-a-1}\|u\|_{a} .
$$

Conversely, assume that $a_{1}<a<a_{2}$, that $u_{j} \in E_{a_{2}}$ and that

$$
\left\|u_{j}\right\|_{b} \leqq M \theta_{j}^{b-a-1} \quad \text { if } \quad b=a_{1} \text { or } \quad b=a_{2} .
$$

By (v) this remains true with a constant factor on the right-hand side if $a_{1}<b<a_{2}$ so the sum $u=\sum \Delta_{j} u_{j}$ converges in $E_{b}$ if $b<a$. Let $E_{b}^{\prime}$ be the set of all sums $u=\sum \Delta_{j} u_{j}$ with $u_{j}$ satisfying (3) and introduce as norm $\|u\|_{a}^{\prime}$ the infimum of $M$ over all such sum decompositions. We have then seen that \|\|$_{a}^{\prime}$ is stronger than \|\|$_{b}$ if $b<a$, while (1) and (2) show that \|\|$_{a}^{\prime}$ is weaker than \|\|$_{a}$. The space $E_{b}^{\prime}$ and, up to equivalence, its norm are independent of the choice of $a_{1}$ and $a_{2}$. In fact, assume that $u=\sum \Delta_{j} u_{j}$ with $u_{j}$ satisfying (3), and let us estimate $\left\|R_{k} u\right\|_{c}$. By (3) and (iv)

$$
\left\|R_{k} u_{j}\right\|_{c} \leqq C M \theta_{k}^{c-a_{v}-1} \theta_{j^{v}}^{a-a-1}, \quad v=1,2 .
$$

We multiply by $\Delta_{j}$ and sum for $j \leqq k$ taking $v=2$ and for $j>k$ taking $v=1$. This gives that

$$
\left\|R_{k} u\right\|_{c} \leqq C M \theta_{k}^{c-a-1} .
$$

Thus the decomposition (1) can be used instead, for any interval. Altogether this shows that the space $E_{a}^{\prime}$ and its topology are independent of the choice of the numbers $a_{1}$ and $a_{2} ; E_{a}^{\prime}$ is defined by (2) for any two values of $b$ to the left and to the right of $a$. (It does not depend on the smoothing operators of course.) In the particular case of Hölder spaces we have $E_{a}^{\prime}=E_{a}$ unless $a$ is an integer.

In (iii) we may replace $\|u\|_{a}$ by $\|u\|_{a}^{\prime}$ if we take another constant, which may tend to $\infty$ as $b$ approaches $a$. In fact, assume we have a decomposition $u=\sum \Delta_{j} u_{j}$ with $u_{j}$ satisfying (3). Then if $b<a_{1}<a<a_{2}$

$$
u-S_{\theta} u=\sum \Delta_{j}\left(u_{j}-S_{\theta} u_{j}\right), \quad\left\|\left(u_{j}-S_{\theta} u_{j}\right)\right\|_{b} \leqq C M \theta^{b-a_{v}} \theta_{j}^{a_{v}-a-1} .
$$

We sum for $\theta_{j}>\theta$ with $v=1$ and for $\theta_{j} \leqq \theta$ with $v=2$ and conclude that

$$
\left\|u-S_{\theta} u\right\|_{b} \leqq C_{b} \theta^{b-a} M
$$

which proves the strengthened form of (iii).

If $u_{k}$ is a bounded sequence in $E_{a}$ for some fixed $a>0$ and $u_{k} \rightarrow u$ in $E_{0}$, it follows from (v) that $u_{k}$ is a Cauchy sequence in $E_{b}$ for every fixed $b<a$ so the limit $u \in E_{b}$. In fact, $u \in E_{a}^{\prime}$ for if we apply (2) to $u_{k}$ and let $k \rightarrow \infty$ it follows that

$$
\left\|R_{j} u\right\|_{b} \leqq C_{a, b} \theta_{j}^{b-a-1} \underline{\varliminf}\left\|u_{k}\right\|_{a} .
$$


We shall say that a sequence $u_{k} \in E_{a}$ is weakly convergent and write $u_{k} \rightarrow u$ in the preceding situation. Note that the definition of $E_{a}^{\prime}$ shows that every element in $E_{a}^{\prime}$ is the weak $E_{a}$ limit of a sequence in $E_{\infty}$.

To state the implicit function theorem we assume that we have another family $F_{a}$ of decreasing Banach spaces with smoothing operators having the same properties as above; we use the same notation for the smoothing operators also. In addition we assume that the embedding $F_{b} \subset F_{a}$ is compact when $b>a$.

Theorem. Let $\alpha$ and $\beta$ be fixed positive numbers, $\left[a_{1}, a_{2}\right]$ an interval with $0 \leqq a_{1}<\alpha<a_{2}, V$ a convex $E_{\alpha}^{\prime}$ neighborhood of 0 and $\Phi$ a map from $V \cap E_{a_{2}}$ to $F_{\beta}$ which is twice differentiable and satisfies, for some $\delta>0$,

$$
\left\|\Phi^{\prime \prime}(u ; v, w)\right\|_{\beta+\delta} \leqq C \sum\left(1+\|u\|_{m_{j}^{\prime}}\right)\|v\|_{m_{j}^{\prime \prime}}\|w\|_{m_{j}^{\prime \prime \prime}}
$$

where the sum is finite. Also assume that $\Phi^{\prime}(v)$, for $v \in V \cap E_{\infty}$, has a right inverse $\psi(v)$ mapping $F_{\infty}$ to $E_{a_{2}}$, that $(v, g) \rightarrow \psi(v) g$ is continuous from $E_{\infty} \cap V \times F_{\infty}$ to $E_{a_{2}}$ and that

$$
\|\psi(v) g\|_{a} \leqq C\left(\|g\|_{\beta+a-\alpha}+\|g\|_{0}\|v\|_{\beta+a}\right), \quad a_{1} \leqq a \leqq a_{2} .
$$

Let $a_{2}$ be at least as large as the subscripts on the right-hand side of (4),

$$
\max \left(m_{j}^{\prime}-\alpha, 0\right)+\max \left(m_{j}^{\prime \prime}, a_{1}\right)+m_{j}^{\prime \prime \prime}<2 \alpha, \text { for every } j ; \alpha-\beta<a_{1} .
$$

For every $f \in F_{\beta}^{\prime}$ with sufficiently small norm one can then find a sequence $u_{j} \in V \cap E_{a_{2}}$ which has a weak limit $u$ in $E_{\alpha}^{\prime}$ such that $\Phi\left(u_{j}\right)$ converges weakly in $F_{\beta}^{\prime}$ to $\Phi(0)+f$.

Proof. Let $g \in F_{\beta}^{\prime}$ and write $g_{j}=R_{j} g$; thus

$$
g=\sum \Delta_{j} g_{j} ; \quad\left\|g_{j}\right\|_{b} \leqq C_{b} \theta_{j}^{b-\beta-1}\|g\|_{\beta}^{\prime} .
$$

We claim that if $\|g\|_{\beta}^{\prime}$ is small enough we can define a sequence $u_{j} \in E_{a_{2}} \cap V$ with $u_{0}=0$ by the recursion formula

$$
u_{j+1}=u_{j}+\Delta_{j} \dot{u}_{j}, \quad \dot{u}_{j}=\psi\left(v_{j}\right) g_{j}, \quad v_{j}=S_{\theta_{j}} u_{j}
$$

and that we have the estimates

$$
\begin{gathered}
\left\|\dot{u}_{j}\right\|_{a} \leqq C_{1}\|g\|_{\beta}^{\prime} \theta_{j}^{a-\alpha-1}, \quad a_{1} \leqq a \leqq a_{2}, \\
\left\|v_{j}\right\|_{a} \leqq C_{2}\|g\|_{\beta}^{\prime} \theta_{j}^{a-\alpha}, \quad \alpha<a \leqq a_{2}, \\
\left\|u_{j}-v_{j}\right\|_{a} \leqq C_{3}\|g\|_{\beta}^{\prime} \theta_{j}^{a-\alpha}, \quad a \leqq a_{2} .
\end{gathered}
$$

Indeed, suppose that $u_{j}$ is already constructed for $j \leqq k$ and that (10), (11) are proved then as well as (9) for $j<k$. We prove (9) for $j=k$ by application of (5) to $v_{k}$ and $g_{k}$ which gives

$$
\left\|\psi\left(v_{k}\right) g_{k}\right\|_{a} \leqq C\left(\theta_{k}^{a-\alpha-1}\|g\|_{\beta}^{\prime}+\theta_{k}^{-\beta-1}\|g\|_{\beta}^{\prime} C_{2}\|g\|_{\beta}^{\prime} \theta_{k}^{\beta+a-\alpha}\right) .
$$


Here we have used the fact that $\beta+a_{1}>\alpha$ by (6). This gives (9) for $j=k$ if $C_{1}>C$ and $\|g\|_{\beta}$ is small, no matter what the value of $C_{2}$ is. (This point is important to avoid circularity in the choice of constants.) Since $u_{k+1}=\sum_{0}^{k} \Delta_{j} \dot{u}_{j}$ we obtain from

$$
\left\|u_{k+1}\right\|_{a}^{\prime} \leqq C^{\prime} C_{1}\|g\|_{\beta}^{\prime}
$$

and we conclude from the strengthened version of (iii) discussed above that (11) is valid for $a<\alpha$ not close to $\alpha$ if $C_{3} / C_{1}$ is large enough. When $a=a_{2}$ the same conclusion is obtained directly by adding (9), and the logarithmic convexity (v) then gives (11) with the same constant in the whole interval. Then we obtain (10) for $j=k+1$ by just summing (9).

We have now proved that the construction of the infinite sequences $u_{j}, v_{j}, \dot{u}_{j}$ is possible, for (12) and (11), with $j=k+1$, show that $u_{k+1}$ and $v_{k+1}$ are in $V$ if $\|g\|_{\beta}^{\prime}$ is sufficiently small. It follows from (9) that $u_{k}$ has a weak limit $u$ in $E_{\alpha}^{\prime}$. What remains is to examine the limit of $\Phi\left(u_{k}\right)$. Write

$$
\begin{aligned}
& \Phi\left(u_{j+1}\right)-\Phi\left(u_{j}\right) \\
& =\left(\Phi\left(u_{j}+\Delta_{j} \dot{u}_{j}\right)-\Phi\left(u_{j}\right)-\Phi^{\prime}\left(u_{j}\right) \Delta_{j} \dot{u}_{j}\right)+\left(\Phi^{\prime}\left(u_{j}\right)-\Phi^{\prime}\left(v_{j}\right)\right) \Delta_{j} \dot{u}_{j}+\Delta_{j} g_{j} \\
& =\Delta_{j}\left(e_{j}^{\prime}+e_{j}^{\prime \prime}+g_{j}\right) .
\end{aligned}
$$

First we estimate

$$
e_{j}^{\prime \prime}=\int_{0}^{1} \Phi^{\prime \prime}\left(v_{j}+t\left(u_{j}-v_{j}\right) ; \dot{u}_{j}, u_{j}-v_{j}\right) d t
$$

by means of (4); our purpose is of course to show that $e_{j}^{\prime}, e_{j}^{\prime \prime}$ are so small that $\Phi\left(u_{k}\right)-\Phi\left(u_{0}\right)$ will have a limit close to $\sum \Delta_{j} g_{j}=g$. If we combine (5) with (9), (10), (11) and recall (6), we obtain, if $\varepsilon>0$ is so small that (6) remains valid if $\varepsilon$ is added in the left-hand side,

$$
\left\|e_{j}^{\prime \prime}\right\|_{\beta+\delta} \leqq C \theta_{j}^{-1-\varepsilon}\|g\|_{\beta}^{\prime 2} .
$$

For any $N>0$ we can choose $\theta_{j}$ so that $\Delta_{j}=O\left(\theta_{j}^{-N}\right)$. For large $N$ we obtain in the same way from Taylor's formula

$$
\left\|e_{j}^{\prime}\right\|_{\beta+\delta} \leqq C \theta_{j}^{-1-\varepsilon}\|g\|_{\beta}^{\prime 2} .
$$

It follows that $\Phi\left(u_{k}\right)$ converges weakly to $\Phi(0)+T(g)+g$ where

$$
T(g)=\sum \Delta_{j}\left(e_{j}^{\prime}+e_{j}^{\prime \prime}\right) .
$$

This sum is uniformly convergent in $F_{\beta+\delta}$ norm when $\|g\|_{\beta}^{\prime}$ is small enough. Hence $T(g)$ is a continuous map from a neighborhood of 0 in $F_{\beta}^{\prime}$ to a compact subset of $F_{\beta}^{\prime}$, and

$$
\|T g\|_{\beta}^{\prime} \leqq C\|g\|_{\beta}^{\prime 2}
$$

By the Leray-Schauder theorem it follows that $g+T(g)$ takes on all values in a neighborhood of 0 , and this proves the theorem. 


\section{References}

[1] Hörmander, L.: The boundary problems of physical geodesy. - Arch. Rational Mech. Anal. $62,1976,1-52$.

[2] NASH, J.: The imbedding problem for Riemannian manifolds. - Ann. of Math. (2)63, 1956, $20-63$.

[3] ZeHNDER, E.: Generalized implicit function theorems with applications to some small divisor problems, I. - Comm. Pure Appl. Math. 28, 1975, 91-140.

University of Lund

Institute of Mathematics

S-220 07 Lund

Sweden

Received 30 March 1984 